\title{
La visión del profesorado de Educación Física sobre la presencia del alumnado de origen extranjero en la escuela: ¿oportunidad oproblema? \\ Physical Education teachers' perspective on the presence of foreign students at school: opportunity or problem? \\ *Gonzalo Flores Aguilar, *Maria Prat Grau, **Susanna Soler Prat \\ *Universidad Autónoma de Barcelona (España), ** INEFC de Barcelona (España)
}

Resumen. Este trabajo analiza las percepciones del profesorado de Educación Física (EF) sobre los efectos y consecuencias que ha generado y/o genera la presencia del alumnado de origen extranjero en su labor profesional y en la dinámica de clase (aula ordinaria y sesiones de EF), además de los motivos que justifican su visión más o menos positiva (oportunidad y/o problema) sobre la misma. Para ello se ha realizado un estudio de casos múltiple con doce docentes que superan los cinco años de experiencia en escuelas públicas multiculturales con más del 50\% de alumnado de origen extranjero. Los instrumentos utilizados para la obtención de datos son las entrevistas semiestructuradas y las observaciones no participantes, y para su interpretación, el análisis de contenido. Entre las conclusiones más relevantes cabe destacar que, según el profesorado de EF participante, dicha presencia no tiene consecuencias negativas en las clases de EF, de la que suelen tener una visión «romántica». En cambio, los efectos sí son percibidos como más negativos en las otras asignaturas impartidas en el aula ordinaria, debido especialmente a los denominados «factores educativos» (falta de personal docente, desigual distribución del alumnado, pocas ayudas de las administraciones públicas, etc.) y a algunas características propias del «perfil de su alumnado» (bajo nivel académico previo, falta de escolarización previa, desconocimiento del idioma vehicular de la escuela).

Palabras clave: Educación física; Escuela multicultural; Pensamiento del profesorado; Alumnado de origen extranjero.

Abstract. This paper analyzes the perspective of Physical Education (PE) teachers on the effects and consequences provoked by the presence of foreign students in their regular and PE classes and in the class dynamics. Also, it aims to understand teachers' reasons about their positive (or negative) perception of classes with foreign students. A multiple case study was conducted with twelve physical education teachers who had more than five years of experience in multicultural public schools (more than 50\% of foreign students). Semi-structured interviews and non-participant observations were used to obtain data, and content analysis was employed to interpret it. As a general conclusion, this study revealed that PE teachers think that the presence of foreign students has no negative consequences in PE classes, which they usually have a «romantic» vision of. However, perceptions are more negative when courses are taught in the regular classroom, especially due to the so-called «educational factors» (absence of teachers, unequal distribution of students, little support of public administrations, etc.) and to some characteristics of the «students' profile» (low previous academic level, lack of schooling, unawareness of the school official language, etc.).

Keywords: physical education, multicultural school, teacher thought, foreigner students.

\section{Introducción}

El progresivo incremento de la diversificación cultural de la población que ha tenido lugar en estas últimas décadas en nuestra sociedad ha generado numerosos debates en torno a los posibles efectos y consecuencias sociales de dicho fenómeno global. Además de la existencia de una visión positiva que concibe a esta nueva realidad social como una oportunidad de convivencia, tolerancia y enriquecimiento cultural (Flecha \& Puigvert, 2002), también se destaca la visión negativa sobre la población inmigrada, la cual se aprecia como una fuente de conflictos (Campoy, 2006), sobre todo por la estigmatización de este colectivo como principal causante de la crisis económica (Cea \& Valles, 2010).

Como cabría esperar, estos discursos también se han trasladado a la escuela. Son muchos los rumores, con menor o mayor volumen, que circulan entre docentes, familiares y estudiantes sobre las particularidades de las escuelas multiculturales, en su gran mayoría, en forma de estereotipos negativos (escuelas conflictivas, bajo nivel académico, etc.) (Aramburu, 2002). Debido a estos prejuicios existentes, los efectos son alarmantes: miedo a trabajar en estas escuelas, huida del alumnado autóctono, etc. (Essomba, 2008).

Esto permite reafirmar el papel de la escuela como marco privilegiado para la inclusión, la socialización y la transformación de la realidad social (Sales, 2007), a partir de la ruptura de estos estereotipos negativos y prejuicios. Cabe recordar que el «desconocimiento cultural» existente entre el colectivo autóctono y el inmigrado, y viceversa, se concibe como uno de los motivos de estas actitudes menos positivas (GarcíaFernández \& Goenechea, 2009). Por este motivo, Benn y Dagkas (2006) y Harrison y Belcher (2006) apuestan por los entornos multiculturales como espacios de acercamiento y descubrimiento mutuo.

De igual manera, el papel del profesorado también adquiere un

Fecha recepción: 28-01-16. Fecha de aceptación: 24-06-16

Gonzalo Flores Aguilar

gonzalo.flores@ub.edu especial interés, aunque la revisión de la literatura deja entrever un panorama preocupante respecto a la manera en la que este colectivo percibe la presencia del alumnado de origen extranjero. Según Aguado, Gil-Jaurena y Mata (2008), suele concebirse como fuente de riqueza en el discurso aparente, pero, en cambio, en la práctica diaria se valora como una situación problemática que puede afectar a su estado anímico personal y laboral (Garreta, 2011), sobre todo por no saber cómo actuar ante los cambios producidos en el aula.

Siguiendo a Flores, Prat y Soler (2014), los tradicionales déficits formativos del profesorado dentro del ámbito de la educación intercultural (EI) y la atención a la diversidad cultural suelen ser la principal causa de esta desorientación en el aula (no saber qué hacer). A su vez, Alegre (2008) alude al «factor educativo» como otro motivo clave que perjudica a la labor del profesorado y a la escolarización del alumnado en escuelas multiculturales, el cual se compone de las siguientes dificultades: a) la distribución desigual de la escolarización del alumnado de origen extranjero (formación de escuelas guetos); b) la falta de personal docente en estas escuelas; c) el poco asesoramiento y ayudas por parte de las administraciones públicas (escuelas olvidadas y segregadas); y d) las continuas entradas y salidas del alumnado debido a la «matrícula viva».

En cuanto al perfil del alumnado de origen extranjero, diversos estudios revelan la existencia de algunas características propias que también suelen afectar negativamente. Entre ellas se encuentran el desconocimiento de la lengua vehicular de la escuela (Aguado et al., 2008; Soriano, 2009); la falta de escolarización o el bajo nivel académico previo (Capllonch, Lleixà \& Godall, 2007; Jordán, 2007); y la poca atención familiar (López-Pastor, Pérez-Pueyo \& Monjas, 2007; Palomero, 2006).

A lo que la EF se refiere, la gran mayoría de estudios realizados coinciden en destacar una visión positiva generalizada entre el profesorado en cuanto a los efectos y consecuencias de la presencia de este alumnado en sus sesiones (Capllonch et al., 2007; Ortí, 2007). Para justificar su opinión, el profesorado piensa que en sus clases «no ha cambiado nada» sobre todo por las «singularidades de la materia» (Flores, 2013): la EF es una asignatura lúdica y práctica, que cuenta con un alumnado muy motivado, y donde la universalidad del lenguaje verbal 
reduce los inconvenientes del desconocimiento del idioma oficial de la escuela (Gil-Madrona \& Pastor, 2003; Prat \& Soler, 2002).

Y es que, como concluyen Soler, Flores y Prat (2012) y Derri, Kellis, Vernadakis, Albanidis y Kioumourtzoglou (2014), la EF puede llegar a tener un gran potencial a la hora de abordar la EI y atender al alumnado de origen extranjero, siempre y cuando haya una intervención docente adecuada.

De hecho, Carroll y Hollinshead (1993) alertan de la existencia de docentes de EF víctimas de la ceguera que les provoca su visión romántica sobre la asignatura, puesto que esta se percibe e interpreta de manera utópica. En este caso, las situaciones conflictivas son naturalizadas o no son «observadas o apreciadas» por este colectivo. Por ejemplo, otros estudios realizados sí que subrayan la existencia de posibles dificultades específicas en la EF que pueden afectar negativamente según el propio profesorado: a) la cultura motriz previa del alumnado, sobre todo la de las chicas; b) el incumplimiento de las normas higiénicas (ducha y ropa deportiva); c) el desconocimiento del idioma oficial de la escuela; y d) las particularidades del alumnado vinculado al Islam (Ramadán, velo, duchas, actividades acuáticas, excursiones, extraescolares y la presión familiar sobre las niñas) (Dagkas, Benn \& Jawad, 2011; López Pastor et al., 2007; Pfister, 2004); entre otras.

En esta línea, en el trabajo de Flores (2013) se detallan un conjunto de limitadores de la EF desde un enfoque intercultural, tradicionalmente obviados por el profesorado, que pueden perjudicar gravemente al alumnado recién llegado: nivel de habilidad motriz; lenguaje verbal y códigos corporales; prejuicios y exclusiones entre el alumnado; contravalores del área; tradición histórica del área; y choques de valores culturales.

Ante este panorama, la mejora de la calidad de la EF desde una perspectiva intercultural, así como también la formación inicial y permanente del profesorado (competencias del profesorado) (Lavega, 2008), requiere de más estudios desde el acercamiento y el contacto real con las escuelas multiculturales y su profesorado (Chepyator-Thomson, You \& Rusell, 2000; Choi \& Chepyator-Thomson, 2011; Dagkas, 2007); sobre todo porque el número de estudios empíricos que se han realizado y/o publicado hasta el momento sobre este campo es escaso y deficitario(Harrison \& Belcher, 2006; López-Pastor, 2012; MacDonald,Abbott, Knez \& Nelson, 2009).

Con la intención de avanzar hacia una EF intercultural como uno de los retos de la EF en el siglo XXI (López-Pastor, Pérez, Manrique \& Monjas, 2016), en este trabajo se le «da la voz» al profesorado de EF para que, en función de sus años de experiencia y sus vivencias, reflexione sobre su labor profesional en un contexto multicultural.

Más concretamente, este estudio pretende conocer: a) la percepción de este colectivo sobre la manera en la que la presencia del alumnado de origen extranjero ha influido o influye en la dinámica del aula y en la propia labor del profesorado, tanto en el aula ordinaria como en las clases de EF y b) los motivos que justifican su visión más positiva (oportunidad) y/o más negativa (problema) sobre la misma.

\section{Metodología}

Esta investigación se enmarca en la perspectiva interpretativa-cualitativa para llevar a cabo un estudio de casos múltiple $(\mathrm{N}=12$ ) (Arnal, Del Rincón \& Latorre, 1994), los cuales han sido seleccionados a partir de un muestreo intencional o de casos típicos (Heinemann, 2003), en

Tabla 1.

Características del profesorado de EF participante

\begin{tabular}{|c|c|c|c|c|}
\hline Escuela/Ubicación & Pseudónimos & Sexo & Edad & Experiencia \\
\hline A. Barcelona. Sant Martí & Laia & $\mathrm{M}$ & 54 años & 13 años \\
\hline A. Barcelona. Sant Martí & Daío & $\mathrm{H}$ & 31 años & 6 años \\
\hline B. Barcelona. Ciutat Vella & Rodrigo & $\mathrm{H}$ & 43 años & 17 años \\
\hline C. Barcelona. Ciutat Vella & Natalia & $\mathrm{M}$ & 36 años & 6 años \\
\hline C. Barcelona. Ciutat Vella & Carmen & $\mathrm{M}$ & 43 años & 12 años \\
\hline D. Barcelona. Ciutat Vella & Lorena & $\mathrm{M}$ & 33 años & 5 años \\
\hline E. Barcelona. Sants & Mar & $\mathrm{M}$ & 43 años & 13 años \\
\hline F. Barcelona. Sants & Ariadna & $\mathrm{M}$ & 34 años & 7 años \\
\hline G. Barcelona. Sant Andreu & Hugo & $\mathrm{H}$ & 39 años & 7 años \\
\hline H. Barcelona. Nou Barris & Álvaro & $\mathrm{H}$ & 34 años & 9 años \\
\hline I. Barcelona. Gràcia & Lucas & $\mathrm{H}$ & 56 años & 15 años \\
\hline J. Sabadell & Mireia & $\mathrm{M}$ & 45 años & 11 años \\
\hline
\end{tabular}

base a dos criterios importantes: a) escuelas públicas de educación primaria con más del $50 \%$ de alumnado de origen extranjero y b) profesorado de EF que supera los cinco años de experiencia laboral en dichas escuelas.

\section{Participantes}

Los doce casos estudiados están formados por cinco hombres y siete mujeres, pertenecientes a diez escuelas diferentes, todas ubicadas en la provincia de Barcelona (ver tabla 1).

\section{Instrumentos}

La entrevista semiestructurada ha sido la primera técnica de recogida de datos utilizada, la cual se caracteriza por su flexibilidad en la formulación de las preguntas, en su mayoría de carácter abierto.

A partir de las directrices de Massot, Dorio y Sabariego (2004), su aplicación ha contado con tres fases diferenciadas. En la «fase de preparación» se concretaron los objetivos de la misma y se elaboró un guión de preguntas. A modo de entrenamiento, en este fase se realizaron tres entrevistas piloto que sirvieron también para la validación de este instrumento (por criterios y por constructo). Tras contactar con las personas participantes, en la «fase de desarrollo» se aplicó el instrumento y se recogió la información extraída a través de un registro de audio. Finalmente, en la «fase de valoración» se estudió la cantidad y calidad de los resultados obtenidos para posteriormente pasar a trascribir literalmente el audio delas doce entrevistas con el apoyo del programa «Express Scribe $\left.{ }^{\circledR}\right)$.

La segunda técnica utilizada ha sido la observación no participante, ya que se ha adoptado un rol de observador externo con la idea de distorsionar lo menos posible la dinámica habitual de las clases (Heinemann, 2003).

Siguiendo las orientaciones de aplicación de Caplow (1972; cfr. Font, 2007), en la «fase previa» también se realizaron diversas pruebas piloto a un profesor no participante en este estudio, pero de características similares, al mismo tiempo que se procedió a la validación del instrumento (por criterios y por constructo). Tras planificar la entrada en el campo de estudio (permisos, horarios, etc.), en la «fase de desarrollo» se utilizaron las notas de campo como registro narrativo (McKernan, 1999). Y como «fase final», se recopilaron, revisaron y clasificaron las sesiones observadas en función del sistema de categorías elaborado.

\section{Análisis de datos}

El instrumento utilizado para el análisis, la codificación y la interpretación de los resultados obtenidos ha sido el análisis de contenido y las fases destacadas por Bardin (1986). En la fase de «preanálisis y preparación del material» se optó por la selección y utilización de todos los materiales producidos por los instrumentos anteriormente descritos, sin excepción. En la fase de «explotación del material» se codificaron los resultados en función del sistema de categorías. Para ello se utilizó la octava versión del programa informático «Nudist NVivo ${ }^{\circledR} »$. Y en último lugar, los informes generados en la fase de «tratamiento de resultados e interpretaciones», según el mismo sistema de categorías que a continuación se detalla, fueron abordados desde una perspectiva cualitativa.

El sistema de categorías utilizado se caracteriza por el proceso deductivo-inductivo empleado para su creación. Es decir, la fundamentación teórica ha permitido la creación deductiva de un primer catálogo de categorías que finalmente ha sido redefinido, inductivamente, a partir del trabajo de campo llevado a cabo.

En definitiva, las categorías desarrolladas que corresponden con la percepción del profesorado de EF estudiado sobre el alumnado de origen extranjero son: a) influencia en la escuela: efectos y consecuencias (labor profesional y dinámica del aula) y b) visión del fenómeno multicultural en la escuela (oportunidad y/o problema).

\section{Resultados y discusión}

A continuación se presentan los resultados obtenidos en función 
del sistema de categorías anteriormente explicado. Para mantener el anonimato de las personas participantes en este estudio se utilizan pseudónimos.

a. Influencia en la escuela: efectos y consecuencias ¿Cómo influye la presencia del alumnado de origen extranjero en el aula ordinaria?

En primer lugar, el profesorado estudiado piensa que existen numerosas diferencias individuales y una amplia gama de niveles educativos entre el alumnado de origen extranjero de una misma clase.

La dificultad es que tútienes muchos niveles de aprendizajes: desde el que te reconoce las letras al que no lee; al que acaba de llegar de Pakistán y no entiende absolutamente nada; o al que llega de la República Dominicana y no ha ido nunca al colegio (Natalia).

Quizás te llega un niño nuevo que a lo mejor no sabe ni coger un lápiz y tú estás haciendo multiplicaciones de dos cifras (Lorena).

Dentro de estos subniveles, la existencia de estudiantes que desconocen la lengua vehicular de la escuela se percibe como un problema, ya que suele suponer la desatención, intencionada o no, de algunos de ellos en el aula.

El tutor yo creo que sí que vive incluso con más frustración y angustia. El tener la problemática de que no esté bien atendido ese niño [...]; y lo ves ahí sentado y dices, incluso se te olvida: «iAy, tengo un niño ahí que solo sabe urdu!». Esa atención genera un estrés de mayor intensidad en los generalistas (Ariadna).

Como señalan Aguado et al. (2008) y Soriano (2009), el idioma es una de las barreras limitadoras más importante para la escolarización del alumnado y para el desarrollo profesional del profesorado que trabaja en el aula ordinaria.

El profesorado participante también denuncia que la «matrícula viva» afecta negativamente al ritmo de trabajo de clase, siendo un motivo más para el descenso del rendimiento académico de todo el alumnado.

En esta escuela es que hay mucha «matrícula viva», es decir, que vienen continuamente. Hay goteo de alumnos que te caen en el mes de enero, te caen en el mes de abril y se encuentran en una escuela que ya lleva una dinámica de todo el curso, y lo tienes que ubicar. Claro, es complicado, porque tú llevas un ritmo (...) Incluso a lo mejor vienen sin ningún conocimiento del castellano (Ariadna).

El rendimiento sería muy bajo. ¡Es que tenemos «matrícula viva»! (Lorena).

Al igual que el profesorado estudiado en Ortiz (2008), en este trabajo se señala a la «matrícula viva» como otro elemento distorsionador de las dinámicas del aula ordinaria, que aumenta y justifica el fracaso escolar, según el profesorado.

A raíz de todas estas dificultades, la carga de trabajo del docente se ve incrementada, hasta el punto de perjudicar gravemente a su estado de ánimo laboral (estrés, presión), como son los casos de Lorena y Mar.

Te afecta. Es que es demasiado. Piensa que a lo mejor tienes tres o cuatro niños que no tienen ninguna necesidad, que son autónomos y que pueden seguir un ritmo normal. iSeis niños de una clase de 25! Los otros, cada uno venido en momentos diferentes, de lugares diferentes, con necesidades completamente diferentes, que uno te ha venido hace cinco meses, el otro tres, el otro te ha venido la semana pasada (...) [Puff] ies para pegarse un tiro! (...)(Lorena). A veces pienso que estar en un cole así es un desgaste (...) es que quieras que no, esto te va chupando la sangre, te va gastando energías y te van saliendo arrugas y canas (Mar).

La existencia de docentes perjudicados a nivel anímico y personal por culpa de su desarrollo profesional en estos contextos ha sido denunciado en otros trabajos previos (Aguado et al., 2008; Garreta, 2011). Erradicar los motivos de origen, en este caso las dificultades con las que se encuentra el profesorado en el aula ordinaria, se antoja una tarea clave para el futuro de la profesión docente y de la educación del siglo XXI.

Como ejemplo, Hugo reafirma la necesidad de contar con más personal educativo en el aula para poder hacer frente a todas las necesidades que se derivan de los grandes subniveles educativos coexistentes en la misma.
Esto supone un desnivel de trabajo para un profesor que no se puede asimilar, es imposible. Tendría que haber cinco profesores, uno para cada alumno (Hugo).

En definitiva, todos los argumentos del profesorado apuntan a que, más allá de la propia diversificación cultural del alumnado, las consecuencias negativas existentes encajan en el «factor educativo» (Alegre, 2008) y en el «perfil del alumnado» (Aguado et al., 2008; Jordán, 2007). Física?

Diez de los doce participantes (83\%) consideran que la presencia del alumnado de origen extranjero en el aula no afecta negativamente ni a la dinámica de la asignatura ni a su labor profesional.

Mi asignatura [EF] es la que menos problemas tiene (Mireia).

Yo pienso que es mucho más difícil en un aula por los subniveles, porque dentro de un aula está el que no sabe leer, el que no sabe multiplicar...y tienes que ir haciendo lo que puedes. En EF bueno, juegan, son niños (...) (Carmen).

Esta percepción positiva también es compartida por el profesorado de EF estudiado en Capllonch et al. (2007) y Ortí (2007).

Entre las razones que justifican esta idea, el profesorado menciona el hábitat de la asignatura (espacio abierto); la dimensión lúdica; los elevados niveles de motivación; y la naturaleza práctica de la asignatura.

Yo creo que hay más dificultades en el aula ordinaria que no en EF. EnEF o te expresas más con gestos, o los niños te ayudan más, yes una actividad, que sí que es de aprendizaje, pero entre comillas más lúdica, y les gusta mucho más a los niños. Están en un espacio abierto y no en un aula cerrada, donde muchas veces no entienden nada de lo que estás explicando (...), y el hábitat, si dijéramos, es diferente ¿no? (Carmen).

A consecuencia de esta naturaleza práctica, una amplia parte de los participantes opina que el lenguaje verbal no es un medio de comunicación tan importante en estas clases. Por este motivo, y a diferencia del profesorado que trabaja en el aula ordinaria, la existencia de estudiantes que no dominan el idioma oficial de la escuela no se considera un problema.

Yo creo que las consecuencias son menores para el profesorado de EF. Aver, yo hablo porque, por ejemplo, hago matemáticas en sexto ytengo a esos niños, y luego los tengo en EF.Al finy al cabo, es bajar al patio y hacer ejercicios (...); solamente con ver, ellos ya intervienen (...) (Mar).

Yo diría que es prácticamente nula [inseguridad del profesor de EF]. Los primeros días no entienden nada, pero como ven que pueden seguir la clase, miran qué hacen los otros (...); la comunicación verbal no sirve de mucho en EF (Rodrigo).

Todos estos motivos coindicen con las «singularidades de la materia» recopiladas en Flores (2013), a partir de los trabajos de Capllonch et al. (2007), Gil-Madrona y Pastor (2003) y Prat y Soler (2002), entre otros.

En el lado contrario, Natalia y Mar sí que revelan la existencia de algunos factores que dificultan el desarrollo de las clases de EF. Contrariamente a lo anteriormente señalado, Natalia afirma que el desconocimiento de la lengua oficial de la escuela conlleva la realización de más demostraciones prácticas en el aula, lo cual afecta a su ritmo de trabajo.

El docente gasta una cantidad de energía impresionante porque no es lo mismo explicar y en cinco minutos tener el juego explicado, $y$ haciendo un ejemploya se entiende. En un centro donde hay tantas culturas diferentes, si hay dos o tres que no entienden nada, tendrás que hacer los ejemplos con ellos, para que los vivencien, que más o menos entiendan de qué va (Natalia).

Además, Natalia afirma que la no escolarización previa de ciertos estudiantes de origen extranjero también puede afectar negativamente; por ejemplo, a la hora de mantener y cumplir unas normas de atención y disciplina.

De repente te llega uno que viene del monte, yentonces lo tienes que educar. Primero no entiende nada, se relaciona pegando, desbarata el funcionamiento del día a día del colegio. Depende si ha sido un 
niño escolarizado o no (Natalia).

De esta misma manera, Mar critica el continuo incumplimiento de las normas higiénicas (indumentaria deportiva) por parte del alumnado extranjero como otro aspecto problemático.

En EF la dificultad está, por ejemplo, en traer el recambio de camiseta. De momento, es que no lo trae ninguno, y llevan aquí ya pues cuatro o cinco meses. Traer el chándal les cuesta [puff], hasta que entienden que esta es la ropa adecuada (Mar).

En el trabajo de López-Pastor et al. (2007) también se recogen las mismas dificultades señaladas por parte de su profesorado de EF estudiado.

En comparación con el discurso más negativo o pesimista que existe entre el profesorado a la hora de mencionar los efectos perjudiciales de la presencia del alumnado de origen extranjero en el aula ordinaria, la visión positiva que posee este mismo colectivo sobre sus clases de EF puede deberse, entre otros motivos, al tradicional discurso romántico existente sobre su materia y sus supuestas singularidades, la cual suele ser considerada una panacea (motiva a todo el alumnado, el lenguaje verbal no es tan importante, etc.) (Flores, 2013).

En este caso, y según Flores, Prat y Soler (2015), este perfil docente (profesorado romántico) se caracteriza por ser un profesional acrítico que no se cuestiona el carácter homogeneizador y etnocéntrico de sus actuaciones (Garreta, 2011; Ortiz, 2008), siendo esto un ejemplo de los procesos de aculturación a los que el alumnado de origen extranjero posteriormente se encuentra en las clases de EF.

A su vez, esta mirada romántica del docente suele provocar la inconsciencia de los ya mencionados aspectos limitadores de la EF desde un enfoque intercultural, tal y como denuncia Flores (2013).

b. Visión sobre el fenómeno multicultural en la escuela Una oportunidad, sí, pero con unas condiciones mínimas

Uno de los principales argumentos que utiliza el profesorado para resaltar la importancia de la diversificación cultural del alumnado hace referencia a la riqueza que supone el hecho de compartir e intercambiar en el aula diversos elementos culturales. En algunos casos se recurre a los juegos del mundo y a otras actividades deportivas en el área de EF, o a otras actividades en las clases de Ciencias Sociales y Lengua Catalana, donde el alumnado de origen extranjero y sus tradiciones y/o experiencias culturales son los protagonistas.

Generalmente se vive de forma enriquecedora, tanto a nivel de convivencia como de aprender cosas nuevas de cada cultura en contacto (Rodrigo).

La diversidad cultural que tenemos te permite trabajar incluso otras cosas como los juegos populares de sus países, etc. y te permite trabajar a lo mejor temas de racismo, que en otros centros síque lo puedes comentar, pero no puedes poner ejemplos digamos tan claros (Darío).

El profesorado estudiado en Capllonch et al. (2007) y Ortí (2007) también afirma realizar proyectos de intercambio interculturales a partir de los juegos y las danzas. En este sentido, cabe recordar que, según el «estadio aditivo» propuesto por Banks (1998; cfr. Essomba, 2008) para el desarrollo curricular multicultural, incorporar en el aula elementos pertenecientes a diferentes identidades étnicas ayuda a reducir la visión etnocéntrica del currículo, lo cual cobra una especial relevancia en un contexto culturalmente heterogéneo.

En segundo lugar, tres profesoras opinan que el contacto directo que se crea en un aula multicultural entre el alumnado de diferentes orígenes y procedencias es un aspecto positivo que, debidamente trabajado, favorece la formación de una sólida competencia intercultural que les capacita para formar parte de esta sociedad diversa. De igual modo, las docentes se muestran convencidas de que estas competencias son menores en el alumnado de otros entornos más monoculturales.

Sí que la considero una oportunidad. Por ejemplo, los niños de sexto son los que menos inmigración tienen, son más autóctonos la mayoría. Estos niños no están tan acostumbrados, como por ejemplo los de tercero, cuarto, o los de parvulario que llevan ya subiendo que hay una barbaridad de multiculturalidad ahí(...). Aellos les cuesta más aceptar a una persona nueva (Mireia).

En otros contextos internacionales, Benn y Dagkas (2006) y Harrison y Belcher (2006) también se muestran convencidos de la necesidad de estos contactos interculturales para la reducción del desconocimiento cultural y los consiguientes estereotipos negativos y prejuicios.

Para que todo ello sea posible, se resaltan unas condiciones mínimas anteriormente ya comentadas: a) el profesorado debe contar con más recursos y personal de apoyo en el aula; b) se deben controlar los desniveles educativos tan extremos que en ocasiones tienen lugar en el aula; y c) el alumnado recién llegado debería haber estado escolarizado previamente.

Totalmente, es una oportunidad (...); lo que pasa es que no tenemos los suficientes recursos como para poder ofrecer esta oportunidad de una manera adecuada y correcta [...] Por ejemplo, este año nos han recortado personal, nos han quitado un profesor; entonces para hacer grupos flexibles en áreas instrumentales como matemáticas y lengua no se pueden hacer, quedan desatendidos (Ariadna).

La diversidad cultural te puede enriquecer (...) si todos tuvieran el mismo nivel de aprendizaje. El problema son los diferentes niveles que tienes (Carmen). tiva

¿Por quées un problema? Justificación de la visión menos posi-

Un amplio porcentaje del profesorado de EF participante (66\%) afirma percibir negativamente el fenómeno multicultural en la escuela (problema), al subrayar todas las dificultades vivenciadas en el aula ordinaria. Además de estas razones que se enmarcan en el ya mencionado «factor educativo» (Alegre, 2008), desde algunos casos se destaca el aumento de actitudes racistas en el barrio.

El problema de esta escuela, y sobre todo de las de este barrio, es que las han dejado convertirse en guetos (Rodrigo).

Un padre viene a la hora de hacer la matrícula (...); puede ver este tipo de alumnado y dirá: ¿yo matricular a este niño? O sea, un padre de nivel bien, social, económico, cultural normal, es un poco reacio a una matrícula aquí(Laia).

Esta última idea coincide con los argumentos de Cea y Valles(2010) y Essomba, (2008), al considerar que la actual crisis económica y el descenso del estado del bienestar ha incrementado el sentimiento de incomodidad y rechazo hacia la población inmigrante, además de la discriminación de las escuelas multiculturales.

En último lugar, las características socioculturales del barrio donde se ubican estas escuelas (barrios pobres y excluidos) y la influencia de la realidad familiar son otras de las razones por las cuales el profesorado piensa que la escuela multicultural es un problema como tal.

Es un problema a la hora de hacer clases, en lo que es en el aula ordinaria. Es un problema porque quieras que no, el barrio es muy importante, en donde estás metido, y con los problemas que el alumnado lleva detrás. Ya no es que culturalmente sean diferentes, porque eso no llega a ser un problema; es la problemática que llevan detrás (incorrecta alimentación, habitan en camas calientes, padres encarcelados, etc.) (Lorena).

Es que la dificultad o el problema no es la diversidad cultural, son las expectativas que tiene cada familia respecto al colegio. Si no te importa en absoluto lo que hagan o dejen de hacer los niños en el colegio (...)(Natalia).

Sobre este último tema, el profesorado estudiado en López-Pastor et al. (2007) y Palomero (2006) también presenta unas percepciones similares al respecto, ya que consideran que las familias inmigradas suelen prestar poca atención y/o falta de interés hacia la escolarización de sus hijos e hijas.

\section{Conclusiones}

Entre el profesorado de EF estudiado existe una clara visión positiva y otra negativa sobre los efectos y consecuencias de la presencia del 
alumnado de origen extranjero en la escuela.

Según este colectivo, los efectos son muy negativos en el aula ordinaria (descenso del ritmo de la clase y del rendimiento académico, aumento de estrés y tensión laboral, etc.), sobre todo por la influencia del «factor educativo» (desequilibrio en la escolarización, falta de recursos, matrícula viva, etc.) y algunas características del «perfil del alumnado» (varios subniveles educativos en el aula, desconocimiento de la lengua materna, no escolarización previa, desatención familiar). Llama la atención como todos los inconvenientes señalados son externos al propio profesorado, lo cual deja entrever una gran falta de autocrítica personal y profesional entre este colectivo.

En el lado opuesto, la mayoría del profesorado de EF (83\%) afirma rotundamente que este alumnado no interfiere negativamente ni en las dinámicas de las clases de EF ni en sus labores profesionales en esta asignatura. A pesar de la complejidad que conlleva poder entender esta respuesta, en este trabajo se alude a la visión romántica de este colectivo sobre la EF como el principal motivo, ya que las limitaciones de la EF desde un enfoque intercultural, entre otros aspectos, pasan desapercibidos para la mayoría del profesorado.

Sin embargo, y a pesar de la situación más bien «favorable» que se encuentra en las clases de EF, más de la mitad del profesorado estudiado (66\%) percibe la presencia general del alumnado de origen extranjero en sus escuelas más como un problema para el que no tienen recursos ni preparación que como una oportunidad. Esta percepción negativa resulta, sin duda, un obstáculo para la EI. Aún así, esta visión no impide que haya, al mismo tiempo también, una cierta valoración positiva por una parte del profesorado participante (34\%), así como el reconocimiento de las oportunidades que conlleva la diversidad cultural (mejora de la competencia intercultural, aumento del intercambio cultural, etc.), lo que denota también la reducida existencia de un discurso ambivalente, favorable a la interculturalidad.

\section{Agradecimientos}

Este estudio ha podido ser realizado gracias a la obtención de una beca de investigación predoctoral de cuatro años de duración concedida al «Departament d’Expressió Musical, Plàstica i Corporal» de la Universitat Autònoma de Barcelona, dentro del programa de Personal Investigador en Formación (PIF) (2009-2013).

\section{Bibliografía}

Aguado, M.T., Gil-Jaurena, I. \& Mata, P. (2008). El enfoque intercultural en la formación del profesorado. Dilemas y propuestas. Revista Complutense de Educación, 19(2), 275-292.

Alegre, M.Á. (2008). Educación e inmigración. ¿Un binomio problemático? Revista de Educación, 345, 61-82.

Aramburu, M. (2002). Los otros y nosotros: imágenes del inmigrante en Ciutat Vella de Barcelona. Madrid: Ministerio de Educación, Cultura y Deporte. Centro de publicaciones.

Arnal, J., del Rincón, D., \& Latorre, A. (1994). Investigación educativa: fundamentos y metodologías. Barcelona: Labor.

Bardin, L. (1986). El análisis de contenido. Madrid: Akal.

Benn, T.C. \& Dagkas, S. (2006). Incompatible? Compulsory mixed-sex physical education initial teacher training (PEITT) and the inclusion of Muslim women: a case-study on seeking solutions. European Physical Education Review, 12(2), 181-200.

Campoy, T.J. (2006). Técnicas cualitativas para la educación intercultural. En A. Pantoja Vallejo \& T. J. Campoy Aranda (Eds.), Programas de intervención en educación intercultural (pp. 51-65). Granada: Grupo editorial universitario.

Capllonch, M., Godall, T. \& Lleixà, T. (2007). El professorat d'educació física a l'escola multicultural. Percepcions del context i necessitats de formació. Temps d'educació, (33), 61-74.

Carroll, B. \& Hollinshead., G. (1993). Etnhicity and conflict in physical education. British Educational Journal, 19(1), 59-76.

Cea, M.Á. \& Valles, M.S. (2010). Evolución del racismo y la xenofobia en España. Informe 2009. Madrid: Ministerio de Trabajo e Inmigración.

Chepyator-Thomson, J.R., You, J. \& Rusell, J. (2000). In service physical education teachers: Background and understanding of multicultural education. Journal of in-Service Education, 26(3), 557-568.

Choi, W. \& Chepyator-Thomson, J. R. (2011). Multiculturalism in teaching physical education: a review of U.S. based literature. Journal of Research, 6(2), 14-20.

Dagkas, S. (2007). Exploring teaching practices in physical education with culturally diverse classes: a cross-cultural study. European Journal of Teaching Education, 30(4), 431-443.

Dagkas, S., Benn, T. \& Jawad, H. (2011). Multiple voices: improving participation of
Muslim girls in physical education and school sport. Sport, Education and Society, 16(2), 223-239.

Derri, V., Kellis, I., Vernadakis, N., Albanidis, E., \& Kioumourtzoglou, E. (2014). The effect of an intercultural Physical Education Program in comparison to the typical one on students' social skills learning. Journal Of Human Sport And Exercise, 9(1), 91-102. Recuperado de: http://www.jhse.ua.es/jhse/article/view/515

Essomba, M.À. (2008). La gestión de la diversidad cultural en la escuela: 10 ideas clave. Barcelona: Graó.

Flecha, J.R. \& Puigvert, L. (2002). Multiculturalismo y educación. En T. Lleixà Arribas, R. Flecha, L. Puigvert, O. Contreras, M. Á. Torralba \& J. Bantulà (Eds.), Multiculturalismo y educación física (pp. 9-45). Barcelona: Paidotribo.

Flores, G. (2013). El profesorado de educación física ante la realidad multicultural: percepción sobre el alumnado, intervención pedagógica y trayectoria profesional. Un estudio de casos. Tesis Doctoral. Universidad Autónoma de Barcelona, Bellaterra. Recuperado de: http://hdl.handle.net/10803/129186

Flores, G., Prat, M. \& Soler, S. (2014). La voz del profesorado de educación física sobre su formación académica ante la realidad multicultural: análisis de la situación y propuestas de mejora. Revista Electrónica Interuniversitaria de Formación del Profesorado,17(2),183-199. Recuperado de: http://www.aufop.com/aufop/ uploaded_files/articulos/1403121378.pdf

Flores, G, Prat, M. \& Soler, S. (2015). La intervención pedagógica del profesorado de educación física en un contexto multicultural: prácticas, reflexiones y orientaciones. Retos. Nuevas tendencias en Educación Física, Deporte y Recreación, (28), 248-255. Recuperado de: http://recyt.fecyt.es/index.php/retos/article/view/35584

Font, R. (2007). La formació permanent del professorat d'educació física de primària i l'educació en valors. Un estudi de casos. Tesis doctoral. Universidad de Barcelona, Barcelona. Recuperado de: http://hdl.handle.net/2445/41477

García-Fernández, J.A. \& Goenechea, C. (2009). Educación intercultural: análisis de la situación y propuestas de mejora. Barcelona: Wolters Kluwer España.

Garreta, J. (2011). La atención a la diversidad cultural en Cataluña: exclusión, segregación e interculturalidad. Revista de Educación, (335), 213-233.

Gil-Madrona, P. \& Pastor, J. C. (2003). Actitudes multiculturales exteriorizadas en educación física: el estudio de un caso y la educación emocional como respuesta. $R e$ vista Complutense de Educación, 14(1), 133-158.

Harrison, L. \& Belcher, D. (2006). Race and ethnicity in physical education. En D. Kirk, D. MacDonald \& M. O'Sullivan (Eds.), Handbook of physical education (pp. 742751). London: Sage Editions.

Heinemann, K. (2003). Introducción a la metodología de la investigación empírica en las ciencias del deporte. Barcelona: Paidotribo.

Jordán, J.A. (2007). Formación intercultural del profesorado de secundaria. ESE. Estudios sobre Educación, (12), 59-80.

Lavega, P. (2008). Educación física y mercado laboral. Competencias profesionales. Cultura, Ciencia y Deporte, 3(8),123-131

López-Pastor, V.M. (2012). Didáctica de la educación física, desigualdad y transformación social. Estudios Pedagógicos, 38,155-176.

López-Pastor, V.M., Pérez-Pueyo, Á. \& Monjas, R. (2007). La atención a la diversidad en el área de educación física. La integración del alumnado con necesidades educativas específicas, especialmente el alumnado inmigrante y de minorías étnicas. Revista digital EFdeportes, (106). Recuperado de: http://www.efdeportes.com/efd106/laatencion-a-la-diversidad-en-educacion-fisica.htm

López-Pastor, V.M, Pérez-Brunicardi, D, Manrique, J.C. \& Monjas, R. (2016). Los retos de la Educación Física en el Siglo XXI. Retos. Nuevas tendencias en Educación Física, Deporte y Recreación, 29,182-187. Recuperado de: http://recyt.fecyt.es/ index.php/retos/article/view/42552/25499

Macdonald, D., Abbott, R., Knez, K. \& Nelson, A. (2009). Taking exercise: cultural diversity and physically active lifestyles. Sport, Education and Society,14, 1-19.

Massot, M.I., Dorio, I. y Sabariego, M. (2004). Estrategias de recogida y análisis de la información. En R. Bisquerra (Ed.), Metodología de investigación educativa (pp. 329-366). Madrid: La Muralla.

McKernan, J. (1999). Investigación-acción y currículum: métodos y recursos para profesionales reflexivos. Madrid: Morata.

Ortí, J. (2007). Educació física i interculturalitat: experiències de l'ef i l'esport relacionades amb els nouvinguts. Trabajo presentado en las Jornades d'Educació Física. CEP, Mallorca. Recuperado de: blocs.xtec.cat/.../files/2011/.../EF-Iinterculturalitat.doc

Ortiz, M. (2008). Inmigración en las aulas: percepciones prejuiciosas de los docentes. Papers, (87), 253-268.

Palomero, P. (2006). Inmigración y educación en la ciudad de Zaragoza: análisis de la realidad y propuestas para una escuela intercultural. Revista Interuniversitaria de Formación del Profesorado, 20(1), 91-111. Recuperado de: http:// www.redalyc.org/pdf/274/27411310005.pdf

Pfister, G (2004). Género y multiculturalidad: la apropiación del cuerpo y la práctica deportiva de las jóvenes inmigrantes. En T. Lleixà Arribas \& S. Soler Prat (Eds.), Actividad física y deporte en sociedades multiculturales: ¿integración o segregación? (pp. 57-80). Barcelona: ICE, Universitat de Barcelona.

Prat, M. \& Soler, S. (2002). Las posibilidades del juego, la actividad física y el deporte para la mejora de la convivencia. Revista Electrónica Interuniversitaria de Formación del Profesorado, 5(2). Recuperado de: http://www.aufop.com/aufop/ uploaded_files/revistas/122843928110.pdf

Sales, A. (2007). La formació dels professionals en educació intercultural. En Grup de Recerca en Educació Intercultural (Ed.), Multiculturalitat, educació $i$ societat (pp. 205-230). Palma de Mallorca: Edicions UIB.

Soler, S., Flores, G., \& Prat, M. (2012). La educación física y el deporte como herramientas de inclusión de la población inmigrante en Cataluña: el papel de la escuela y la administración local. Pensar a Prática, 15(1), 1-19. Recuperado de: https:// revistas.ufg.emnuvens.com.br/fef/article/view/16653

Soriano, E. (2009). La formación del profesorado para construir una nueva sociedad. En E. Soriano (Ed.), Vivir entre culturas: una nueva sociedad (pp. 53-83). Madrid: La Muralla. 